\title{
AOR
}

Selected Papers of \#AoIR2021:

The 22nd Annual Conference of the

Association of Internet Researchers

Virtual Event / 13-16 Oct 2021

\section{HOW TO BE ALGORITHMICALLY GOVERNED LIKE THAT: DATA- AND ALGORITHMIC AGENCY FROM USER PERSPECTIVE}

Author \#1 Ana Pop Stefanija

Author \#1 imec-SMIT, Vrije Universiteit Brussel

Author \#2 Jo Pierson

Author \#2 imec-SMIT, Vrije Universiteit Brussel

Our social lives are being increasingly governed by algorithmic, artificial intelligence (Al) and automated decision-making (ADM) systems. If users are being the subject of the governance by algorithms (Just and Latzer, 2017), and companies and regulators have been proposing ways for governance of algorithms (Saurwein, Just and Latzer, 2015), what role is there for the user? Often put aside, this third actor in the tripartite network of technology-regulation-user(s), almost doesn't have a say in if and how this governance is taking place. With this paper, we aim to tackle this issue. We are interested in a third type of governance - where users have also governing power(s) over algorithmic systems.

Our main research question is how do we enable users to actively govern algorithms, instead of passively being governed by them? And what do the users need in order to be algorithmically governed in such a way that will enable for more agency, autonomy and control when interacting with Al systems and being an object of their algorithmic outputs.

We take the theoretical conceptualizations about algorithmic governance (Katzenbach and Ulbricht, 2019; Latzer and Festic, 2029; Introna, 2016) and the related notion of algorithmic governmentality (Bellanova, 2017; Rouvroy 2013, 2020) as a starting point for discussing the state of how it is so we arrive to what ought to be. Algorithmic governance is becoming a pervasive form of (co-)governing that affects and influences users' behavior by steering actions, and limiting and influencing choices (Latzer and Festic, 2019), affecting users' autonomy and agency. It is understood as a form of social ordering that governs (by shaping, enabling and constraining activities) (Latzer and Festic, 2019)) by relying "on coordination between actors, is based on rules and

Suggested Citation (APA): Pop Stefanija, A. and Pierson, J. (2021, October). How to be algorithmically governed like that-data- and algorithmic agency from user perspective. Paper presented at AolR 2021: The 22nd Annual Conference of the Association of Internet Researchers. Virtual Event: AolR. Retrieved from http://spir.aoir.org. 
incorporates particularly complex computer-based epistemic procedures." (Katzenbach and Ulbricht, 2019, p. 2). This notion is closely related with that of algorithmic governmentality, understood as "government of the social world that is based on the algorithmic processing of big data sets rather than on politics, law, and social norms" (Rouvroy, 2020; see also Rouvroy, 2013 and Bellanova, 2017). As Beer (2017) outlines - algorithmic systems participate in a kind of a social ordering of the world, having a "constitutive or performative role in ordering that world on our behalf." (p.4). Since users are being governed without real knowledge, control, agency and autonomy over both the algorithmic processes and the data collections enabling them, we aim to sketch a possible path for reversing the imbalanced power positions.

In order to investigate what is it that users need to actively participate into governance of algorithms, we designed and conducted a participatory technographic (Bucher, 2012) research with 47 participants. We opted for a guided and supportive process where participants were able to reflect on the process, formulate and elaborate their insights, thoughts, needs, and requirements based on their lived experience, i.e., after a real interaction with these algorithmic systems. Through a guided multi-stage process, consisting of taking a survey, filing a Subject Access Request (Article 15 of the General Data Protection Regulation) and purposeful interaction with the Transparency tools of the platform of their choice (Facebook, Instagram, Twitter, Spotify, Netflix, Tinder, Google and TikTok), during a period of three months, the participants provided us with a series of outputs. One of the outputs, in a form of pre-structured diary, required them to compile a list of requirements for agency and trust. We used these outputs as inputs for our analysis.

We focus on agency because it is a prerequisite for a "power to" as an opposite of "power over", characteristic for algorithmic governmentality. Our results show that agency is preconditioned by three elements: to be provided with information, to be able to gain knowledge and to be afforded with ability(/ies). The first two are foundational elements that will enable individuals to act/have agency. These abilities range from having control over data flows and cycles, to influencing algorithmic outputs and acting with autonomy and self-reflection.

We translate the requirements of ability to see, know and act into 3 main principles the "power to" requires the elements of (data-, outputs-, self-) sovereignty, transparency and explainability. Transparency, or ability to access to information, is the first important, but not sufficient element. Explainability, or the opportunity and ability to gain knowledge, should provide individuals with agential power, ability to act and thus, to (re)gain sovereignty.

These requirements and principles should be implemented at either interface only or both interface and infrastructure level. These leads to what we call agency affordances. Agency affordances are potentialities for action, where the possibility to act with agency is coupled with the ability to act. While the aim of embedding interface agency affordances via elements (located in specific buttons and features (Bucher and 
Helmond, 2018) is to promote and make agency visible, agency affordances need to be "programmed" at system level (to make agency doable) via functions, enabling agency through different dynamics, conditions, and practices.

When programming and embedding agency affordances, it is important to keep in mind that they require the coming together of various actors, processes, infrastructures, and levels, happening both before and after the development and employment of $\mathrm{Al}$ systems. As such, their implementation is context, user, and system dependent. And since exercising agency also requires not just the possibility, but also the knowledge of how to act and of ability to act, attention should be dedicated to improving literacy and skills efforts. However, to mitigate overburdening the individual, regulation should play supporting, by crucial part too.

References:

Beer, D. (2017). The social power of algorithms. Information, Communication \& Society, 20(1), 1-13. https://doi.org/10.1080/1369118X.2016.1216147

Bellanova, R. (2017). Digital, politics, and algorithms: Governing digital data through the lens of data protection. European Journal of Social Theory. https://doi.org/ $10.1177 / 1368431016679167$

Bucher, T. (2012). Programmed sociality: A software studies perspective on social networking sites. 221.

Bucher, T., \& Helmond, A. (2018). The Affordances of Social Media Platforms. In The SAGE Handbook of Social Media (pp. 233-253). Sage Publications. https://dare.uva.nl/ search?identifier=149a9089-49a4-454c-b935-a6ea7f2d8986

Hummel, P., Braun, M., Augsberg, S. and Dabrock, P. (2018). Sovereignty and Data Sharing. ICT Discoveries, 2, p. 1-10.

Introna, L.D, (2016). Algorithms, Governance, and Governmentality: On Governing Academic Writing. Science, Technology, \& Human Values, 41(1), 17-49.

Just, N. \& Latzer, M. (2017). Governance by Algorithms: Reality Construction by Algorithmic Selection on the Internet. Media, Culture \& Society, 39(2), 238-258.

Katzenbach, C. \& Ulbricht, L. (2019). Algorithmic governance. Internet Policy Review, 8(4). DOI: 10.14763/2019.4.1424 
Latzer, M. \& Festic, N. (2019). A guideline for understanding and measuring algorithmic governance in everyday life. Internet Policy Review, 8(2).

https://doi.org/10.14763/2019.2.1415

Rouvroy, A. (2020). Algorithmic Governmentality and the Death of Politics. Green European Journal. https://www.greeneuropeanjournal.eu/algorithmic-governmentalityand-the-death-of-politics/

Rouvroy, A. and Berns, T. (2013). Algorithmic governmentality and prospects of emancipation. Réseaux, 177(1), 163-196.

Saurwein, F., Just, N. and Latzer M (2015) Governance of Algorithms: Options and Limitations. The journal of policy, regulation and strategy for telecommunications, information and media, 17 (6), 35-49.

Thornton, L., Knowles, B., and Blair, G. (2021). Fifty Shades of Grey: In Praise of a Nuanced Approach Towards Trustworthy Design. Conference on Fairness, Accountability, and Transparency (FAccT'21), March 3-10, 2021, Virtual Event, Canada. ACM, New York, NY, USA, p. 64-77. https://doi.org/10.1145/3442188.3445871 